\title{
Discussion on Development of Lu Zhe Chengni Inkstone
}

\author{
Yongmei Wu \\ Shandong College of Arts \\ Jinan, China 250001
}

\begin{abstract}
As one of the four famous inkstones of China, Chengni inkstone's popularity is nowhere near Duan inkstone, She inkstone and Tao inkstone. Besides, Chengni inkstone suffers many mishaps, from making a noise to declining, stopping firing, lose of technology and archaeology and afterburning. During the intermittent process, Chengni inkstone fails to develop well. Until today, under the support of national intangible cultural heritage, new research on and propulsion of development of Chengni inkstone appear in various places. Lu Zhe Chengni inkstone is uniquely owned by Zhegou town, Sishui county, Shandong province. It has strong local features and it is worth deeply researching.
\end{abstract}

Keywords-Lu Zhe Chengni inkstone; development; innovation

\section{INTRODUCTION}

"It becomes a distinguished guest on desk after grinding, and large bell form comes out of runner. If the sound runs through to spread outward, seven times of summoning arouse resonance in people's heart." Emperor Qianlong describes collected bell-type Lu Zhe Chengni inkstone in this way. Lu Zhe Chengni inkstone is Tao inkstone. Purifying is a kind of technology for making Tao inkstone and mud is the material for making a inkstone. Just as its name implies, Chengni inkstone is made by using clarified mud to make an inkstone blank and then roasting. Chengni inkstone is different from Duan inkstone, She inkstone, Tao inkstone and other stone inkstones. Its making and roasting have strong operability. In 2007, Lu Zhe Chengni inkstone is listed as intangible cultural heritage of Shandong province. Mr. Yang Yuzhen, director of $\mathrm{Lu}$ Zhe Chengni Inkstone Institute in Zhegou town, Sishui county, Shandong province, is inheritor of intangible cultural heritage, promoting heritage and development of Lu Zhe Chengni inkstone to some degree. However, the power of one person is limited. According to investigation, due to current $\mathrm{Lu}$ Zhe Chengni inkstone's similar imitation, conformism, backward craft and so on, it is usually regarded as "miscellaneous inkstone of low-grade" and fails to win attention. If Lu Zhe Chengni inkstone aims to develop, it needs to find itself and change current situation.

\section{CurRent Development Status of Lu Zhe Chengni INKSTONE}

As production place of Lu Zhe Chengni inkstone, Zhegou town, Sishui County, Shandong borders on Qufu, Shandong- hometown of Confucius and it has excellent cultural tradition. Pottery making in Zhegou area has a history of at least 5000 years. Generations of pottery makers are cultivated here and they have wide consensus and internal enthusiasm towards development and research of Chengni inkstone. In late 1980s and early 1990s, under the support of local government in Shandong, Mr. Shi Ke, a famous inkstone specialist and sealing cutting specialist, and Mr. Yang Yuzhen organize burning experiments in Zhegou town, Sishui county and achieve success in 1991. It attracts general attention from scholars and inkstone lovers both at home and abroad. When traveling in Japan, $\mathrm{Gu} \mathrm{Mu}$, vice president of national Chinese People's Political Consultative Conference at that time, gives $\mathrm{Lu}$ Zhe Chengni inkstone to Japanese as a national gift. Besides, he also gives it to chief of government of Korea, Singapore, Thailand, Germany, Italy, America and so on, officials of the United Nations and friends in Hongkong and Taiwan area. However, 25 years have past and research and development of $\mathrm{Lu}$ Zhe Chengni inkstone has no great progress "see "Fig. 1". According to field investigation, there are several Lu Zhe inkstone factories in Zhegou town, Sishui County with Chengni workshop and manufacturing shop and without product research and development department. Inkstone manufacturing adopts gypsum mould to aid in forming and pattern part is completed by handmade fine graving. In addition, there are villagers organizing production in their own courtyard. Kiln furnace is simple steamed bun kiln and coal is used for burning. Local inkstone makers in Zhegou town all have a "inkstone manual", which is almost the copy of the same version. Inkstone is mostly made according to modeling and pattern on inkstone manual. Mr. Shi Ke is born in 1924 and he is 91 years old. Mr. Yang Yuzhen, director of Lu Zhe Chengni inkstone institute in Zhegou town, Sishui County, is nearly 80 years old. They make great contributions to after-burning of Lu Zhe Chengni inkstone. But they are too old to be able to exert their ambition in innovative research and development of Lu Zhe Chengni inkstone. When chatting with local people about innovative research and development of $\mathrm{Lu}$ Zhe Chengni inkstone, they think that it is very necessary and approve it. But they do not know how to do and what to do. 


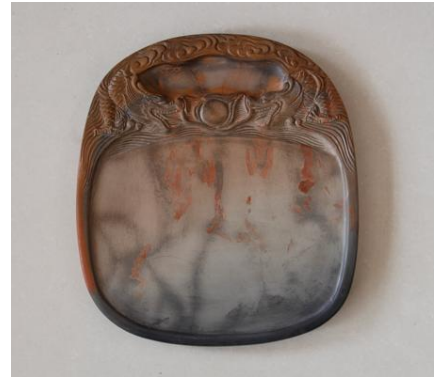

Fig. 1. Lu Zhe Chengni inkstone of the contemporary era.

\section{Mud FEATURES OF LU ZHE CHENGNI INKSTONE}

Development of $\mathrm{Lu}$ Zhe Chengni inkstone requires deepening research on raw materials, understanding its mud property and perfecting its manufacturing craft. Raw material of $\mathrm{Lu}$ Zhe Chengni inkstone is exclusively owned by Zhegou town, Sishui County, Shandong province with high quality. It is worth researching deeply, developing and exploiting. Primary soil used in Lu Zhe Chengni inkstone is the most precious gift to local by nature. Just like Yixing clay in Dingshu town, Yixing, it is exclusively owned by this area. Production of Chengni inkstone mainly centers on Shanxi, Henan, Shandong and so on. Raw material of Shanxi Chengni inkstone is Fenshui mud and that of Henan Chengni inkstone is Yellow River mud. In terms of ceramic raw materials, they are both regeneration soil. Only raw material of Lu Zhe Chengni inkstone in Shandong is primary soil and it is uniquely owned by Zhegou area. According to collected Zhegou soil data: In the place 0 to 3 meters from the earth's surface, storage capacity of Zhegou soil ore is about 1.22 billion cubic meters. It has high viscosity with extremely fine soil texture. According to colors, Zhegou soil can be divided as the five colors of white, red, yellow, reddish brown and black (as in "Fig. 2") with small dilatation coefficient and thermostability. These data are shallow for research on mud features of Lu Zhe Chengni inkstone. Deeper data analysis and contrast with other pugs (such as Yixing clay and kaolin) are required.

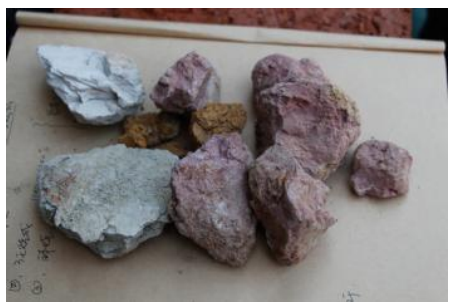

Fig. 2. Green-ore made of Zhegou earth in different colors

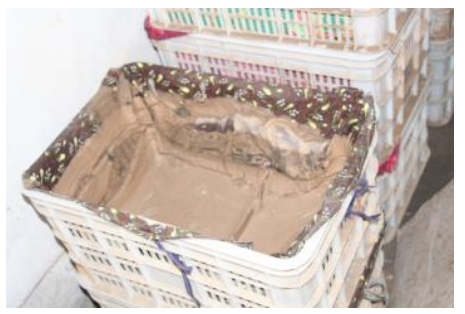

Fig. 3. Purified mud.
After natural mouldiness, drying in the sun, soaking in water and purifying mud, Zhegou soil ore can be used to make an inkstone. Grain of purified mud is an important difference between Chengni inkstone and other inkstones. During the process of purifying mud, overlay two or several different local colors in Zhegou and vertical section will have natural and beautiful grain after purifying mud, just like twisted colored body(as in "Fig. 4"). It can imitate stone grain or wood grain. But what is the most important is that it imitates nothing and just tries to be itself. If there is scientific data analysis on pug, operations of research on purified mud in the labor will be simple and effective. Many effects obtained by chance will become rational and will not be that mysterious. What's more, unique-styled mud purifying effect of $\mathrm{Lu}$ Zhe Chengni inkstone can be achieved.

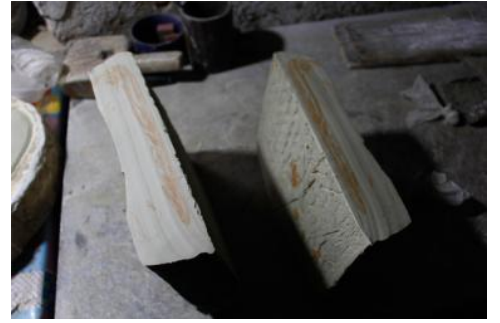

Fig. 4. Vertical section of purified mud

\section{INNOVATIVE DEVELOPMENT OF LU ZHE CHENGNI INKSTONE}

Progress can only be achieved by changing and development can only be achieved by innovation. Innovative sense is the key to development of old things. Lu Zhe Chengni inkstone is also the same. According to current development situation and mud features of Lu Zhe Chengni inkstone, this paper will discuss development of Lu Zhe Chengni inkstone in the following several aspects.

\section{A. Craft Innovation}

Primary soil mineral used in inkstone manufacturing in Zhegou is unique with high quality. It will be a shame if we fail to make Chengni inkstone demonstrating features of mud material and it will be a waste of raw material. Manufacturing of Lu Zhe Chengni inkstone in Zhegou area still uses craft of 1990 s, which is basically the same as mud purifying craft in Shanxi and Henan. Excavated mud raw ore (as in "Fig. 2") usually is not treated with purifying to make an inkstone instantly. It is firstly stored in an old woven bag for more than 6 months. After natural mouldiness, put it in the sun to dry and raw ore naturally smashes into powder without extra processing. Put Zhegou soil experiencing sieving and removal of impurities into a mud purifying sump. After soaking in water for one night, mud submerges at the bottom. Use a water pump to swab-off water on the surface of mud purifying sump and put mud material into a white pass box coated by percale to naturally sink (as in "Fig. 3"). During the process of mud sinking, exposure in the sun is forbidden and it needs to naturally get dry inside a room whose temperature is above $0^{\circ} \mathrm{C}$. It takes more than 3 months to complete natural mud sinking, depending on climate. Well purified mud has no 
bubbles in its vertical section and there is no need to pug before making an inkstone. It is reasonable that these traditional techniques are still used. They are summary of wisdom of previous generations and they are relatively suitable for small manual workshop. If constant temperature drying rooms constructed according to mud features of $\mathrm{Lu}$ Zhe Chengni inkstone are available, mud purifying time will be shortened and work efficiency will be enhanced.

Nowadays, inkstone manufacturing in Zhegou area usually uses plaster mold. After carving an inkstone, many plaster molds will be made through duplication and they are similar to mold moulding of domestic ceramic. Cut purified mud into needed sizes, put them in plaster molds and use a mallet to hit it hard as in "Fig. 5". After plaster mold absorbs all the water on the surface of mud inkstone, you can take mud out of the mold. Then, the general modeling of an inkstone is completed. It only requires to conduct finer finishing on pattern as in "Fig. 6". At the same time of saving labor time, plaster mold produces completely same works, which lack handmade beauty. Inkstone belongs to scholar wares. Originally, it is a tool for scholars and painters to do painting and calligraphy. Apart from having the function of providing ink, it needs to satisfy people's need of playing to some degree. With gradual improvement of people's living standard, people's need in spiritual aspect gets higher and higher. It is extremely difficult for Lu Zhe Chengni inkstone shaped in plaster mold to squeeze into the list of high quality inkstone. Many modern ceramic molding methods may be helpful to manufacturing of $\mathrm{Lu}$ Zhe Chengni inkstone and they are worth learning and referring to. Traditional craft is sedimentary deposit of culture and it is worth inheriting. But we cannot follow the beaten track. Craft innovation of $\mathrm{Lu}$ Zhe Chengni inkstone requires enhancing inkstone maker's acceptance ability and learning ability of new things.

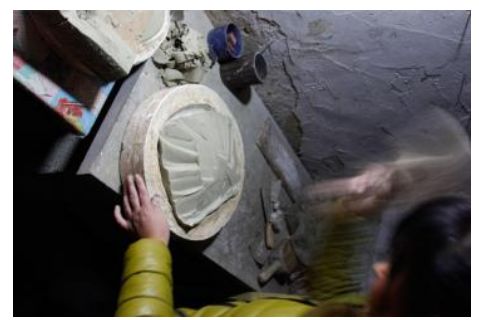

Fig. 5. Moulage for mud purifying

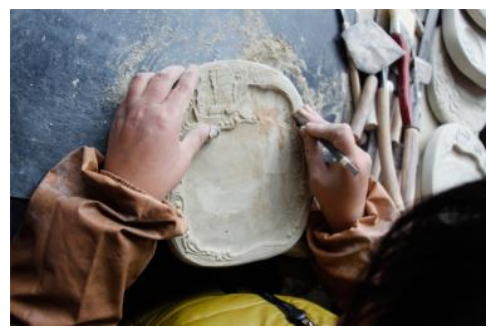

Fig. 6. Fine and exquisite finishing

\section{B. Modeling Innovation}

As stated above, Lu Zhe Chengni inkstone lacks innovation of modeling. Most modelings, patterns and sizes come from inkstone manual, leading to same style in the market and making it easy for buyers to have aesthetic fatigue. We often hear that somebody's one design increases sale greatly and saves a ceramic factory which is dying. It is sufficient to prove the importance of modeling design. Local inkstone makers in Zhegou directly face consumers and realize that current consumers like exquisite modelings better. In order to cater to market and consumers, they also have innovative sense. But their innovation is mainly piecing together of existed modeling or using new patterns for reference instead of real innovation.

In terms of modeling innovation of $\mathrm{Lu}$ Zhe Chengni inkstone, firstly we need to determine aimed people, then determine usage of inkstone. You design an inkstone for children, adults or old people? Is it used to do painting or for appreciation? Nowadays, many children learn calligraphy and need inkstones. Children's inkstone cannot be too heavy and its volume should be much smaller than normal size. Adults' inkstone tends to be practical with concise and elegant modeling and without too many cravings, leaving more space as center of inkstone. Compared with other people, old people have more spare time and only things with cultural connotation can enter their eyes. Old people's inkstone should not only have relatively stable modelings, but also have proper modeling decoration. Inkstone used for writing and painting usually emphasizes on practicability. It should be convenient for writing. In terms of modeling design, user's feeling should be considered. For example, many men of literature and writing pursue returning to innocence. Purified mud does not always uses plaster mold. Instead, it uses a wooden stick to pat Chengni clod in an emotional and random way, grasps the moment of form and processes ink pond and inkstone center according to formal tendency. Inkstone made in this way is natural and random, but it has high requirements for maker's accomplishment in several aspects. If you make modeling design for $\mathrm{Lu}$ Zhe Chengni inkstone for the purpose of appreciation, it is required to inject more layers and connotations of appreciation into Chengni inkstone. For example, peach-shaped inkstone of Tang dynasty adopts the beautiful implied meaning of longevity of birthday peaches. Modeling innovation of $\mathrm{Lu}$ Zhe Chengni inkstone requires applying craft according to material based on service requirements.

\section{Firing Innovation}

Before firing of Lu Zhe Chengni inkstone, there is an important process, namely drying as in "Fig. 7". Trimmed inkstones with similar drying degree should be put together and green body of different drying degrees put together will arouse cracking. It should be placed inside a room where there is no wind to dry in the shade. Inkstone is solid green body and exposure to the weather can arouse excessively rapid shrinking of surface and lead to cracking in green body. Generally speaking, it takes at least more than 1 month and at most 3 months to fire in a kiln after completion of inkstone's green body, depending on climate. This problem can also be solved with the help of modern technology. An oast with constant temperature can reduce drying time and reduces cracking of green body due to uneven shrinking during drying. 


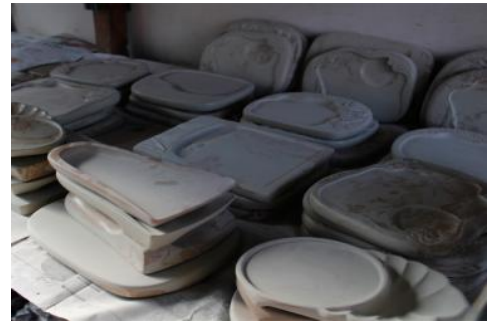

Fig. 7. Finished inkstone taking shelter from the wind and drying in the shade

So far, Lu Zhe Chengni inkstone stills uses original semiconductivity flame earth kiln to fire (as in "Fig. 8"). It uses coal as fuel and firing time is 5 to 7 days. Green body of inkstone is put into a sagger after complete drying. Kiln placing worker bends to pass through the kiln gate which is about 1 meter high and move it inside the kiln as in "Fig. 9" and starts to burn-in with soft fire after sealing kiln gate. Local earth kiln has two chimneys. Front chimney is straight flame used to expelling water in green body, which takes 2 to 3 days. When free water between molecule of inkstone's green body is completely drained, the front chimney can be sealed, the back chimney can be opened and using semi-conductivity flame principle of steamed bun kiln can be used for firing. Generally, there is smoulder things in the sagger holding inkstones, such as wheat stem, saw powder and so on. When certain temperature is reached, smoulder things automatically burn the inkstone, producing smoulder effect and fallen ash effect of plant ash during firing. Firing temperature of Lu Zhe Chengni inkstone in Zhegou town, Sishui county, Shandong province is $900^{\circ} \mathrm{C}$ or so, belonging to low temperature non-glazed pottery. During firing process, burning spot appears due to inadequate restoration, which is furnace transmutation broadcast frequently in the market. Earth kiln has a long firing time and coal firing is not environmental friendly. Can modern gas shuttle kiln be used to fire Lu Zhe Chengni inkstone? It is worth researching and conducting experiments. In 2015, Huang Yuying, a teacher and a Taiwan ceramist gives a lecture about her smoulder works in Shandong. Her firing method of using silver paper to wrap metal is worth referring to and imitating. In addition, many foreign methods of salt burning and soda burning can be referred to. Traditional earth kiln firing just puts inflammable in the sagger. What will be the effect if metallic oxide is put in the sagger for firing? It needs constant experiments and deep research. Only by mastering furnace transmutation and firing of $\mathrm{Lu}$ Zhe Chengni inkstone scientifically, can Chengni inkstone of different colors be produced through firing according to shape and structure, or can multiple colors be produced from one shape through firing to achieve perfect unification of shape, quality and color.

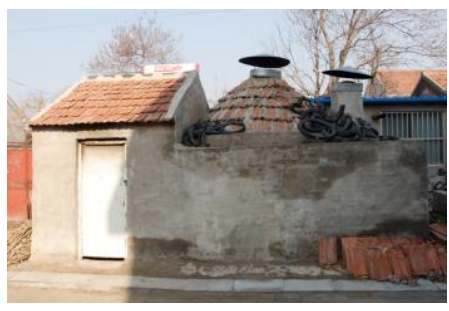

Fig. 8. Earth kiln

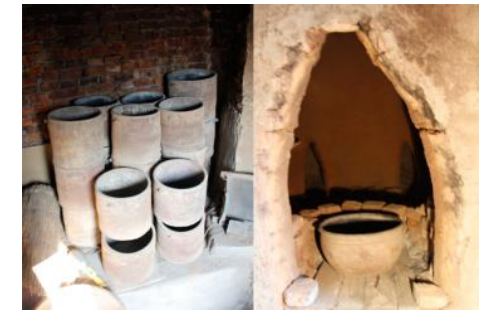

Fig. 9. Sagger and kiln eye of earth kiln

\section{CONCLUSION}

Current development of $\mathrm{Lu}$ Zhe Chengni inkstone needs fresh "blood", bold innovation and bold try. It requires scientific analysis on mud material, improving mud purifying method, designing novel appearance molding, researching firing curve carefully, improving kiln properly and enhancing rate of finished products of firing. At the same time, different fillers need to be selected according to modeling and pattern of inkstone to obtain different firing results.

\section{REFERENCES}

[1] Shi Ke, Lu Zhe Chengni inkstone, Qingdao press,1996.

[2] Mi Fu, Inkstone History and Inkstone Manual, China Bookstore, 2014.

[3] Yang Yuzhen, Pottery Village Documentary, Jining Press and Publication Bureau, 2000.

[4] Yan Zuan, Inkstone. Shandong Fine Arts Press, 2007.

[5] Lin Tao, Chengni Inkstone, Hunan Fine Arts Press, 2010.

[6] Shen Xiaoxiao, Chinese Chengni Inkstone Craft Research. Doctoral Thesis.

[7] You Xiaoman, Artistic Research on Chengni Inkstone Shape and Structure Design. Master's Thesis. 